\title{
Maxwell-Wagner Relaxation in Ca-, Sm- and Nd-doped Ceria
}

Rida Ahmed, ${ }^{1}$ Shuting Wang, ${ }^{2}$ Sajid ur Rehman, ${ }^{3}$ Jie Sun, ${ }^{4}$ Jin Wang, ${ }^{2}$ Renjun $\mathrm{Si},{ }^{2}$ Ankang Zhu, ${ }^{5}$ Yi Yu, ${ }^{2}$ Qiuju Li ${ }^{2}$ and Chunchang Wang $^{2,6, *}$

\begin{abstract}
Doped ceria, i.e. $\mathrm{Ce}_{1-x} \mathrm{M}_{\mathrm{x}} \mathrm{O}_{2-\delta}$ with $\mathrm{M}$ being dopant metal, has been a focus of great attention for solid oxide fuel cells (SOFCs) due to their high oxygen conduction. In the past literature, the dielectric relaxations in these materials have been ascribed to be caused by defect associates (MCe"-Vö) possessing different $\mathrm{MCe}^{\prime \prime}$ and $\mathrm{V} \ddot{o}$ distances. But we believe that with changing measurement and analysis techniques, it is necessary to invest our time to re-examine the already reported materials and take a detailed investigation of the underlying phenomenon behind their dielectric relaxations again. Thus, we have used solid-state reaction to prepare $\mathrm{Ce}_{1-\mathrm{x}} \mathrm{M}_{\mathrm{x}} \mathrm{O}_{2-\delta}$ with $\mathrm{M}=\mathrm{Ca}, \mathrm{Sm}$, and $\mathrm{Nd}$ in $\mathrm{x}=0.1,0.2$, and 0.3 ratios, respectively. The as-prepared and post annealed samples were tested for dielectric properties from 300-1080 K with varying frequencies. The lowtemperature relaxation (R1) was argued to be a Maxwell-Wagner relaxation caused by humidity sensitivity. The hightemperature relaxation (R2) was ascribed to be caused by the hopping motion of oxygen vacancies. This fact was also supported by a detailed analysis of impedance spectra. While according to the previous reports, this relaxation is because of the oxygen-vacancy-dopant defect pair.
\end{abstract}

Keywords: Dielectric; Maxwell-Wagner relaxations; Defects; Impedance.

Received: 12 January 2021; Accepted date: 30 March 2021.

Article type: Research article.

\section{Introduction}

Ionic conducting oxides, especially ceria and its compounds, have acquired fame for the manufacture of solid oxide fuel cells, oxygen sensors and electrochemical applications..$^{[1-4]}$ Different dopants have been added to the ceria matrix according to the required applications in the past literature. The properties of ceria vary remarkably with different dopants and their concentrations. Although extensive literature can be found on the effect of the desired dielectric properties with specific dopants in ceria, most of the work select the dopant to be 1) either a single element 2) or multiple elements belonging

${ }^{1}$ Institute of Solid State Physics, Chinese Academy of Sciences, Hefei 230031, PR China.

${ }^{2}$ Laboratory of Dielectric Functional Materials, School of Physics \& Material Science, Anhui University, Hefei, 230601, P. R. China. ${ }^{3}$ High Magnetic Field Laboratory, Chinese Academy of Sciences, Hefei 230031, Anhui, P. R. China.

4 State Key Laboratory of ASIC \& System, School of Microelectronics, Fudan University, Shanghai 200433, China.

${ }^{5}$ Institutes of Physical Science and Information Technology, Anhui University, Hefei 230601, P. R. China

${ }^{6}$ State Key Laboratory of Low-Dimensional Quantum Physics, Department of Physics, Tsinghua University, Beijing 100084, P. R. China.

*Email: ccwang@ahu.edu.cn (C. Wang) to the same group (hence same valence state and properties) of the periodic table..$^{[5-6]}$ Also, among it, about every other work is on the Lanthanides series. ${ }^{[6]}$ This conventional selection of dopants gives rise to the questions in the mind of the reader: are lanthanides the only suitable elements for ceria doping? Will the dielectric permittivity change with the change of dopants? Would the nature of the dielectric relaxations alter with the change of dopant?

In the present work, we have selected one alkaline earth element (Ca) and two $3 \mathrm{~d}$ transition elements ( $\mathrm{Sm}$ and $\mathrm{Nd}$ ) as dopants so a clear understanding can be made about the nature of the dielectric relaxations. Ceria $\left(\mathrm{CeO}_{2}\right)$ is a fluoritestructured compound showing ionic (oxygen) conduction. The Ce ion exists in a tetravalent state and when is doped with lesser valent ions, such as di-valent $\left(\mathrm{M}^{2+}\right)$ and tri-valent $\left(\mathrm{M}^{3+}\right)$ dopants, charge imbalance is created in the fluorite structure, which is compensated with the generation of oxygen vacancies $\left(\mathrm{V}_{\mathrm{O}}\right){ }^{\left[{ }^{[5]}\right.}$ These vacancies become mobile with the elevation of temperature making ceria a good oxygen-ion conducting electrolyte. Because of this factor, it has one of the major applications as an electrolyte in solid oxide fuel cells..$^{[6]}$ A single divalent dopant ion produces one oxygen vacancy, whereas it takes two trivalent dopants to generate a single vacancy. ${ }^{[7-12]}$ In the past work, it has been reported that mostly the dopant ions and oxygen vacancies associate to form $\mathrm{MV}_{\mathrm{O}}$ 
pairs and isolated $\mathrm{M}$ ion carrying an effective positive and negative charge respectively and rarely associate to generate neutral $\mathrm{M}_{2} \mathrm{~V}_{\mathrm{O}}$ triplet. ${ }^{\left[{ }^{13,14]}\right.}$ According to Kroger-Vink notation:

$$
\mathrm{M}_{2} \mathrm{O}_{3} \rightarrow\left(\mathrm{M}_{\mathrm{Ce}} \mathrm{V}_{\mathrm{o}}\right)+\mathrm{M}_{\mathrm{Ce}}^{\prime}+3 \mathrm{O}_{\mathrm{O}}^{\mathrm{x}} \text {. }
$$

These $\mathrm{MV}_{\mathrm{O}}$ pairs are considered to be responsible for creating dielectric dipoles that give rise to Debye relaxations in the doped ceria. ${ }^{[15,16]}$

There are reports where the dielectric behaviour and relaxations for pure ceria are the same as those of doped one. ${ }^{[17]}$ It means that $M V_{O}$ pairs cannot be the only reason for this anticipation stimulated us to perform a detailed dielectric study on doped ceria to verify the origin of relaxation peaks. $\mathrm{Ca}, \mathrm{Sm}$ and $\mathrm{Nd}$ have been used as dopants for ceria, and their dielectric behaviour has been analyzed. ${ }^{[17]}$ Dopants ratio in ceria have been varied, and a comparison is made to which percentage of an element gives the highest value of dielectric response. Yamamura et al. have prepared $\mathrm{Ce}_{1-\mathrm{x}} \mathrm{Ca}_{\mathrm{x}} \mathrm{O}_{2-\delta}, \mathrm{Ce}_{1-}$ ${ }_{\mathrm{x}} \mathrm{Sm}_{\mathrm{x}} \mathrm{O}_{2-\delta}$ and $\mathrm{Ce}_{1-\mathrm{x}} \mathrm{Nd}_{\mathrm{x}} \mathrm{O}_{2-\delta}$ with ratios varying from 0.1 to 0.9 and have studied their dielectric properties. ${ }^{[18-20]}$ where the values of $0.1,0.2$ and 0.3 gave the highest permittivity values for $\mathrm{Ca}, \mathrm{Sm}$ and $\mathrm{Nd}$, respectively. However, they have ascribed both of the relaxations to be caused by defect associates $\left(\mathrm{MCe}^{\prime \prime}-\mathrm{V} \ddot{o}\right)$ possessing different $\mathrm{MCe}^{\prime \prime}$ and $\mathrm{V} \ddot{o}$ distances. Whereas, we believe that the high-temperature relaxation maybe a Maxwell-Wagner relaxation. Thus, in this works we have prepared $\mathrm{Ce}_{0.9} \mathrm{Ca}_{0.1} \mathrm{O}_{2-\delta}, \mathrm{Ce}_{0.8} \mathrm{Sm}_{0.2} \mathrm{O}_{2-\delta}$ and $\mathrm{Ce}_{0.7} \mathrm{Nd}_{0.3} \mathrm{O}_{2-}$ $\delta$ by solid-state reaction and a detailed analysis of the dielectric properties of the as-prepared and oxygen annealed samples has been performed in order to analyze the true nature of the relaxations.

\section{Experimental section}

\subsection{Synthesis Procedure}

$\mathrm{CeO}_{2}$ (99.9\% Aldrich co.) was taken as the starting material. $\mathrm{CaCO}_{3}$ (99.9\% Aldrich co.) $\mathrm{Sm}_{2} \mathrm{O}_{3}$ (99.9\% Aldrich co.) and $\mathrm{Nd}_{2} \mathrm{O}_{3}$ (99.9\% Aldrich co.) were doped in 10 wt.\%, 20 wt.\% and $30 \%$ with respect to ceria. The powders were mortared for about 60 minutes by hand until they were completely mixed. The powders were then calcined at $800{ }^{\circ} \mathrm{C}$ for $2 \mathrm{~h}$ and $1000^{\circ} \mathrm{C}$ for $5 \mathrm{~h}$. Ca was completely doped in ceria while $\mathrm{Sm}$ and $\mathrm{Nd}$ didn't react completely with ceria and hence were again heated for $10 \mathrm{~h}$ at $1500{ }^{\circ} \mathrm{C}$. Single phased $\mathrm{Ce}_{0.9} \mathrm{Ca}_{0.1} \mathrm{O}_{2-\delta}(\mathrm{CCO})$, $\mathrm{Ce}_{0.8} \mathrm{Sm}_{0.2} \mathrm{O}_{2-\delta}(\mathrm{CSO})$ and $\mathrm{Ce}_{0.7} \mathrm{Nd}_{0.3} \mathrm{O}_{2-\delta}(\mathrm{CNO})$ were prepared as can be evidenced by X-ray diffraction (XRD) analysis.

Rectangular pellets of uniform thickness $(1.5 \mathrm{~mm})$ were made for $\mathrm{CCO}, \mathrm{CSO}$ and $\mathrm{CNO}$ with the addition of PVA. The pellets were first sintered at $600{ }^{\circ} \mathrm{C}$ for $2 \mathrm{~h}$ to ensure the complete removal of PVA and then were sintered at $1600{ }^{\circ} \mathrm{C}$ for $10 \mathrm{~h}$.
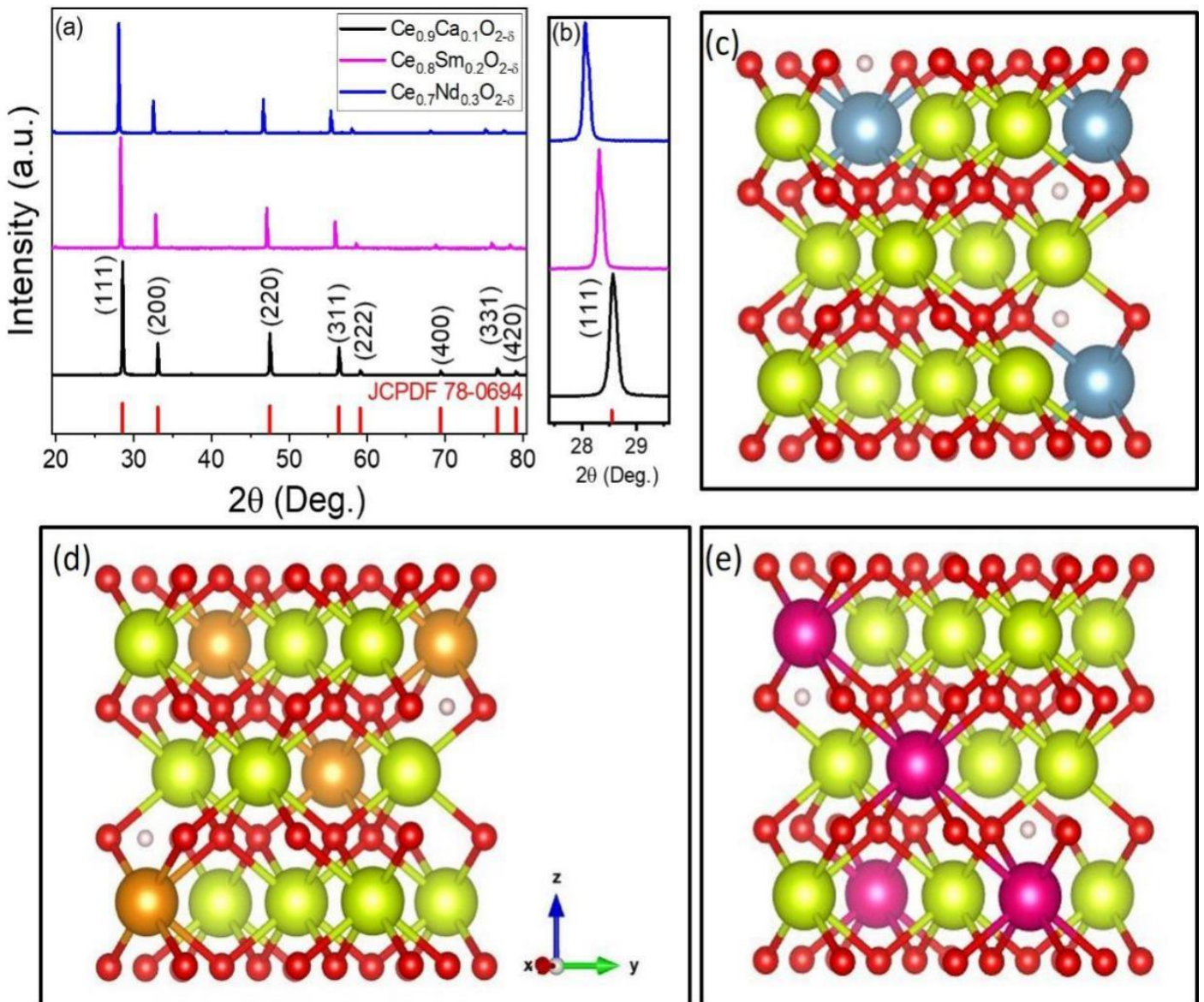

Fig. 1 (a) XRD patterns of the as-prepared pellets of CCO, CSO, and CNO. The vertical lines are of pure $\mathrm{CeO}_{2}(\mathrm{JCPDF} 78-0694)$. (b) The focused (111) peak showing a shift in $2 \theta$ with dopant concentration. Ball-and-stick representations of the cubic fluorite structure of (c) CCO, (d) CSO and, (e) CNO. 

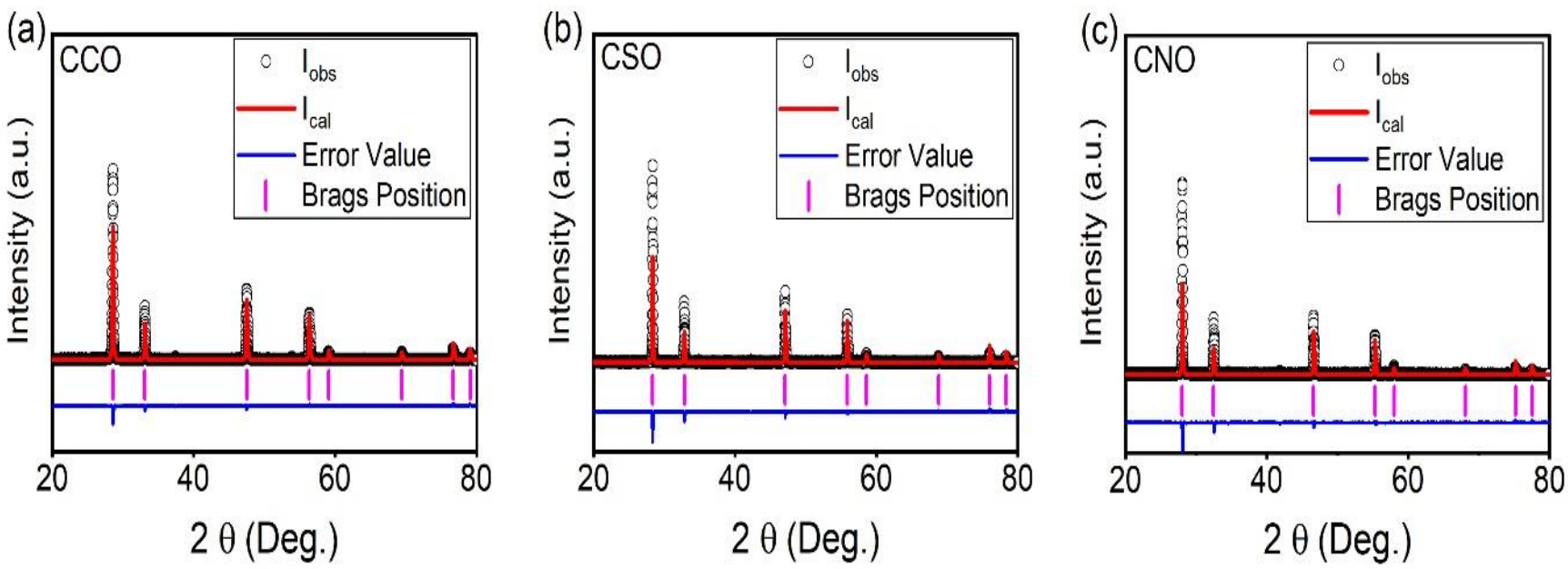

Fig. 2 The Rietveld refinement XRD patterns of (a) CCO, (b) CSO and (c) CNO.

\subsection{Characterization}

XRD analysis was performed by Smart Lab diffractometer (Rigaku Smartlab Beijing Co, Beijing, China) with $\mathrm{Cu} \mathrm{K} \alpha$ radiation. A field emission scanning electron microscope (SEM, Model S-4800, Hitachi Co., Tokyo, Japan) was used to study the morphology of the pellet surfaces. Dielectric measurements and impedance analysis were performed in high temperature range from room temperature to $800{ }^{\circ} \mathrm{C}$ by a Wayne Kerr 6500B precise impedance analyser (Wayne Kerr Electronic Instrument Co., Shenzhen, China).

\section{Results and discussions}

Fig. 1(a) shows the XRD patterns of the $\mathrm{CCO}, \mathrm{CSO}$, and $\mathrm{CNO}$ with reference to the pure ceria peaks (JCPDF 78-0694). We can see that with the increase of dopants ratios, the peak positions have shifted towards the left side of the reference line (Fig. 1(b)). The continuous shift in the peaks with the change of dopant concentration $(\mathrm{Ca}=10 \%, \mathrm{Sm}=20 \%$, and $\mathrm{Nd}=30 \%)$ shows that the increase in dopant concentration causes distortion in the lattice structure. Also, no impurity peaks are observed, indicating that all the samples are single phase. The Rietveld refinement XRD of the samples is shown in Fig. 2. By careful examination, the peaks matched perfectly with Bragg's position peaks (shown in pink) with error values (shown in blue), indicating that no impure phase of ceria exists in any of the samples. The details of the XRD parameters, an average of crystallite size and lattice constant are calculated by as shown in Table 1. It can be seen that lattice constant is continuously increasing with the change of dopants. This is because of the distortions created by doping of heavier atoms in place of $\mathrm{Ce}$ ions in the matrix. Ceria, having a cubic fluorite

Table 1. XRD characterization data of CCO, $\mathrm{CSO}$ and CNO.

\begin{tabular}{llll}
\hline Parameters & CCO & CSO & CNO \\
\hline Crystal system & cubic & cubic & cubic \\
Space group & Fm- $3 \mathrm{~m}$ & Fm-3m & Fm- $3 \mathrm{~m}$ \\
Lattice constant & $\mathrm{A}=5.413 \AA$ & $\mathrm{a}=5.433 \AA$ & $\mathrm{a}=5.463 \AA$ \\
Crystallite Size & $55.4 \mathrm{~nm}$ & 67.45 & 69.71 \\
\hline
\end{tabular}
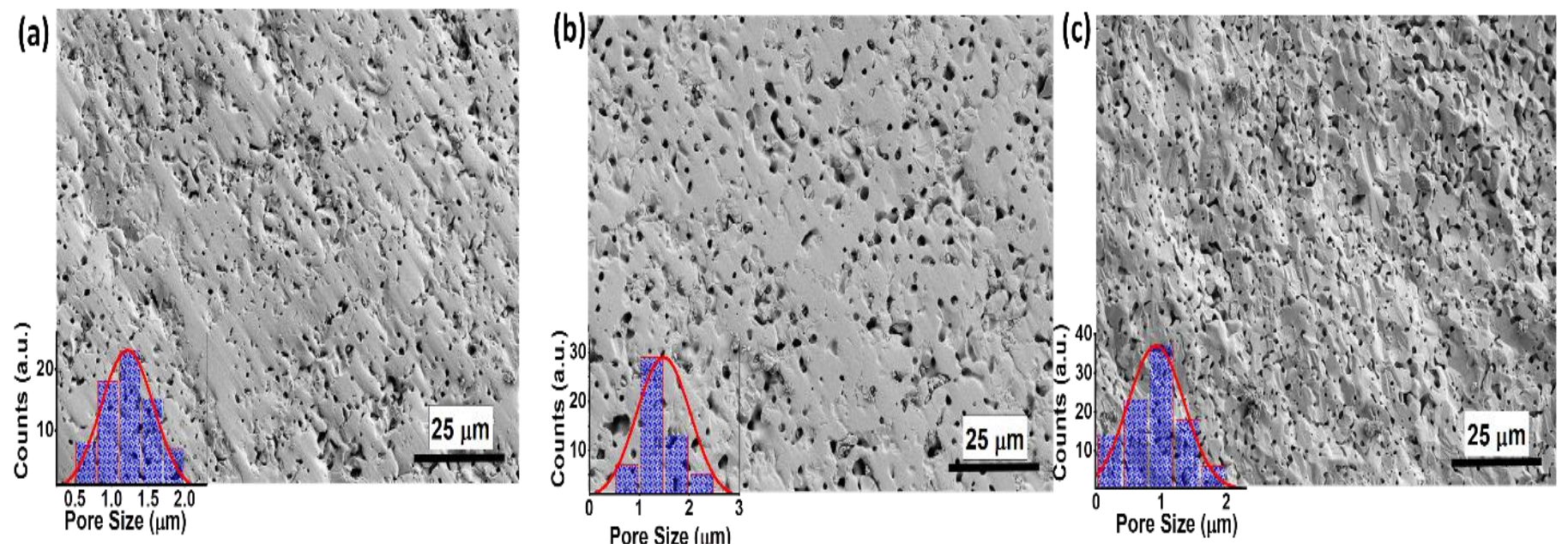

Fig. 3 The SEM images of (a)CCO, (b)CSO, and (c)CNO. The insets show the calculation of pore size in the form of histograms through standard deviation for each sample. 
structure, belongs to $F m \overline{3} m$ space group. This implies that it has four $\mathrm{CeO}_{2}$ formula unit per cell. The unit cells have high symmetry with lattice parameter $\mathrm{a}=5.411 \AA$. The $\mathrm{Ce}$ and $\mathrm{O}$ atoms occupy $(0,0,0)$ and $(1 / 4,1 / 4,1 / 4)$ atomic sites. In an undoped ceria structure, a single $\mathrm{Ce}$ atom is surrounded by eight $\mathrm{O}$ atoms on the cubes' edges. Thus, when dopant atoms are introduced into the lattice matrix, oxygen vacancies will be generated. ${ }^{[2]]}$ Fig. 1(c-e) shows the lattice structures (created by VESTA-JP-Minerals) of $\mathrm{Ca}, \mathrm{Sm}$ and $\mathrm{Nd}$ doped ceria with oxygen vacancies generated as a result of dopant atoms.

Fig. 3 shows the SEM images of the as-prepared pellets. The images show the polished and thermally etched crosssection of the pellets. The pore size is calculated through standard deviation by SEM images and shown in histograms in insets. The average pore size is almost the same for all three samples, as shown in the insets of Fig. 3. However, we can see that the average pore count is maximum for $\mathrm{CNO}$ and least for $\mathrm{CCO}$. The generation of pores is justifiable as the replacement of $\mathrm{Ce}^{3+}$ ions (ionic radius $\sim 0.97 \AA$ ) with dopants of higher ionic radii $(\mathrm{Ca} \sim 1.12 \AA, \mathrm{Sm} \sim 1.079 \AA$ and $\mathrm{Nd} \sim 1.109 \AA)$ generates tensile stress in the samples. To reduce the stress, it is energetically favourable for the samples to create pores
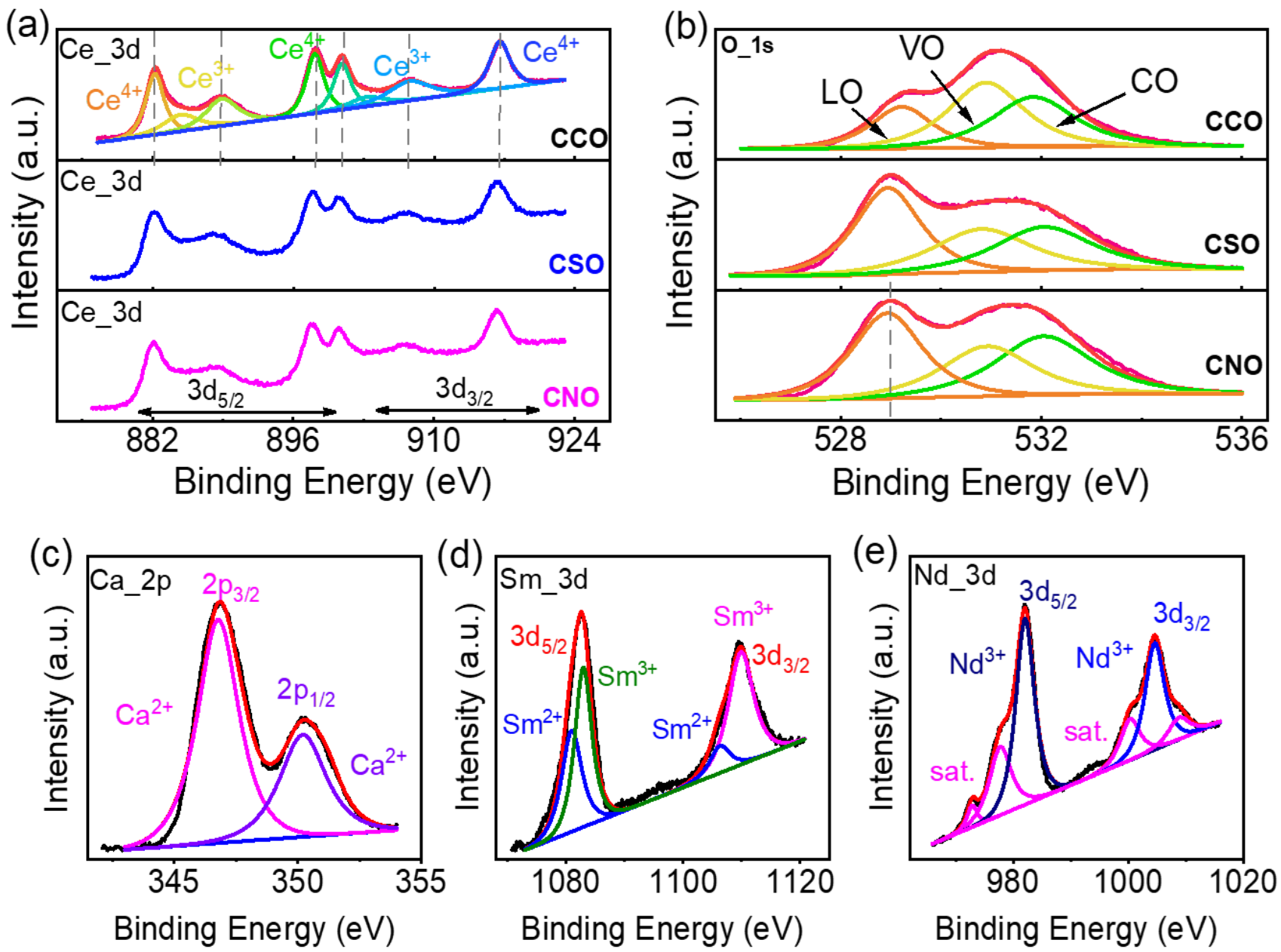

Fig. 4 The XPS images of (a) Ce ions and (b) O ions of the as-prepared samples. Ca ion concentration in $\mathrm{CCO}(\mathrm{c}), \mathrm{Sm}$ ion concentration in $\mathrm{CSO}(\mathrm{d})$, and $\mathrm{Nd}$ ion concentration in $\mathrm{CNO}(\mathrm{e})$. 


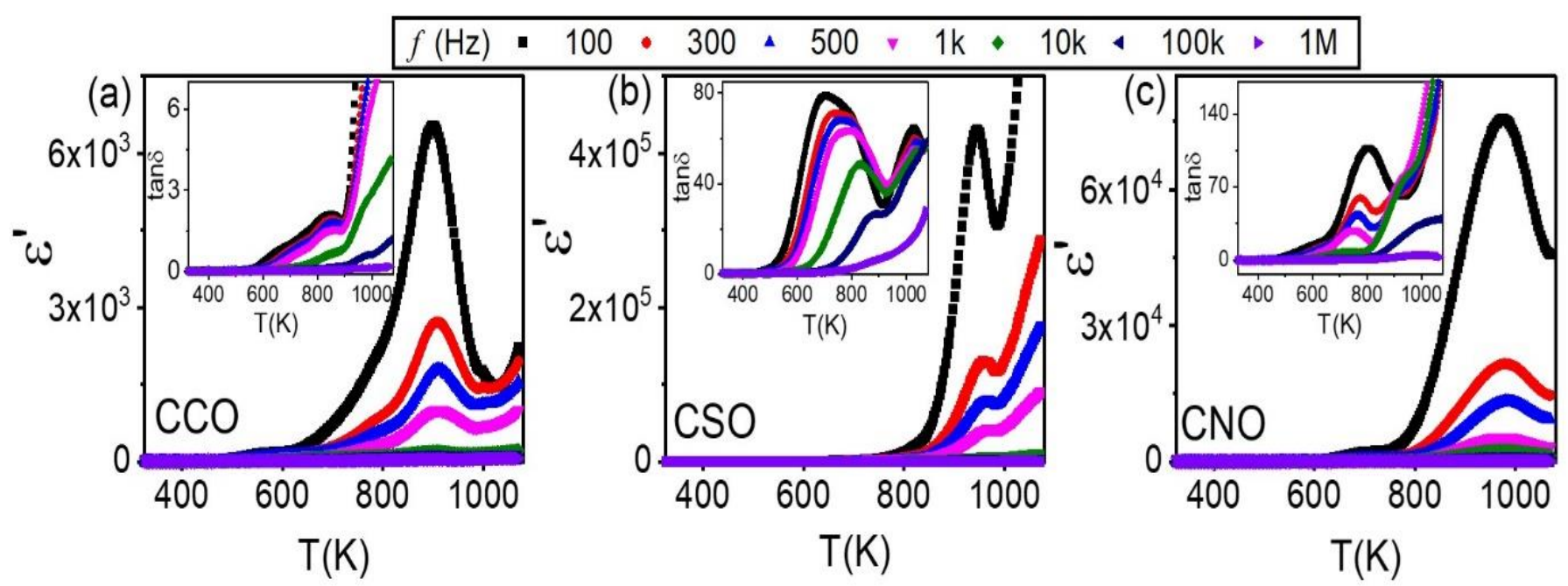

Fig. 5 Temperature dependent permittivity and tangent loss (inset) for as-prepared (a) CCO, (b) CSO, and (c) CNO recorded with different frequencies.

higher temperature, whose peak position changes with the increase in frequencies. A noticeable shift in peak positions is seen towards higher temperature for all three samples with the increase in frequency. This shows that the relaxations are thermally activated. Relaxation time $(\tau)$ plays a vital role in thermally activated relaxations as it dominates their peak positions. Peaks in the curves are obtained at a specific frequency when $\omega \tau=1$ and then begins to decrease with the increase in temperature. The relaxations in all the samples seem shadowed. To remove the background's shadowing, the imaginary part of the electric modulus $\mathrm{M}^{\prime \prime}(\mathrm{T})$ is usually calculated as a function of temperature and plotted to reveal the relaxation phenomenon.

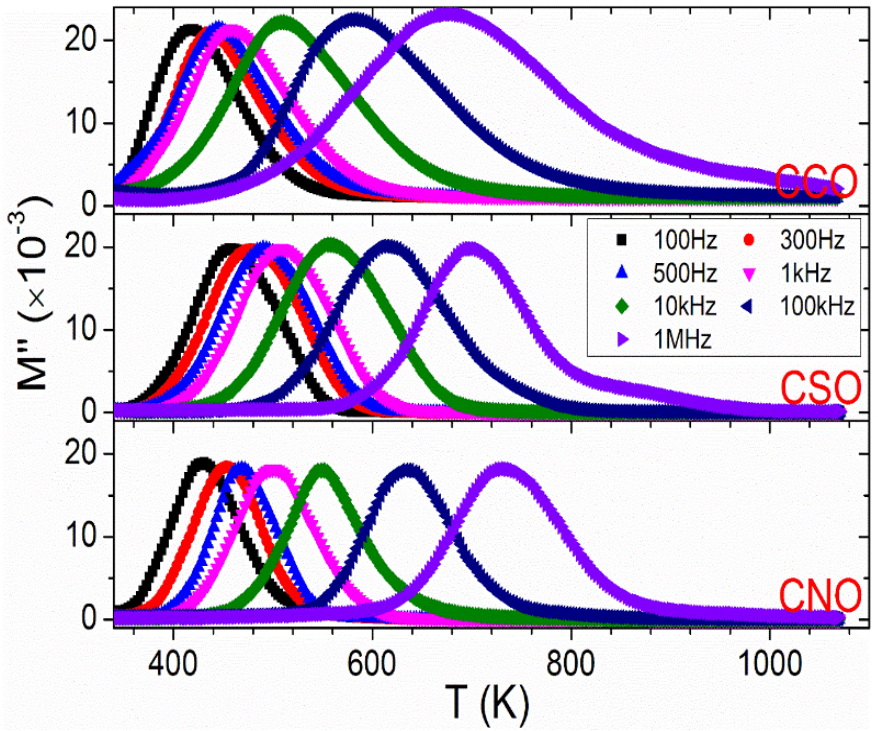

Fig. 6 Temperature dependence of the modulus spectra for $\mathrm{CCO}, \mathrm{CSO}$, and $\mathrm{CNO}$ recorded with different frequencies.

The temperature-dependent dielectric properties of the asprepared $\mathrm{CCO}, \mathrm{CSO}$, and $\mathrm{CNO}$ samples were investigated in terms of electric modulus spectra and summarized in Fig. 6. The electric modulus spectra were used because the oxygen- ion conducting electrolytes frequently exhibit notable conductivity, especially in the temperature higher than room temperature. This conductivity can yield remarkable increasing background that shadows the dielectric relaxation. In this case, the electric modulus, defined as $M *=M^{\prime}+$ $j M^{\prime \prime}=1 / \varepsilon *(M$ 'and $M$ "are the real and imaginary parts of $M *, j=\sqrt{-1}$ ), can effectively eliminate the background. ${ }^{[19]}$

At first glance, Fig. 6 shows one set of thermally activated relaxation peaks for all samples. However, a careful examination reveals that the peaks are composed of two close relaxation processes. To shed light on these relaxations, the curves were fitted using two Gaussian peaks to detach the peaks. As an example, the fitting results of $\mathrm{CCO}$ were displayed in Fig. 7, and the fitting results of $\mathrm{CSO}$ and $\mathrm{CNO}$ were given in Fig. S1 and S2. Perfect agreement between the experimental data (points) and the fitting results (solid curves) are achieved, indicating that the samples possess two thermally activation relaxation processes. ${ }^{[32]}$ For brevity, the low-and high-temperature relaxations are named as $R_{1}$ and $R_{2}$, respectively.

Table 2. Relaxation parameters for $\mathrm{CCO}, \mathrm{CSO}$, and $\mathrm{CNO}$.

\begin{tabular}{ccccc}
\hline \multirow{2}{*}{$\begin{array}{c}\text { Sampl } \\
\mathrm{e}\end{array}$} & $\begin{array}{c}E_{\mathrm{a}} \\
(\mathrm{eV})\end{array}$ & $f_{0}(\mathrm{~Hz})$ & $E_{a}(\mathrm{eV})$ & $f_{0}(\mathrm{~Hz})$ \\
\hline $\mathrm{CCO}$ & 0.83 & $2.40 \times 10^{12}$ & $1.05 / 0.60$ & $8.47 \times 10^{13} / 4.57$ \\
& & & & $\times 10^{9}$ \\
$\mathrm{CSO}$ & 1.02 & $2.09 \times 10^{13}$ & 0.99 & $1.11 \times 10^{12}$ \\
$\mathrm{CNO}$ & 0.83 & $3.98 \times 10^{11}$ & 0.96 & $1.69 \times 10^{12}$ \\
\hline
\end{tabular}

Based on the fitting results, the peak positions can be accurately deduced. The measurement frequency $(f)$ was plotted as a function of the peak position $\left(T_{P}\right)$, according to the Arrhenius law:

$$
f=f_{0} \exp \left(-E_{a} / k_{B} T\right)
$$

where $f_{0}$ is the pre-exponential factor, $E_{a}$ is the activation 

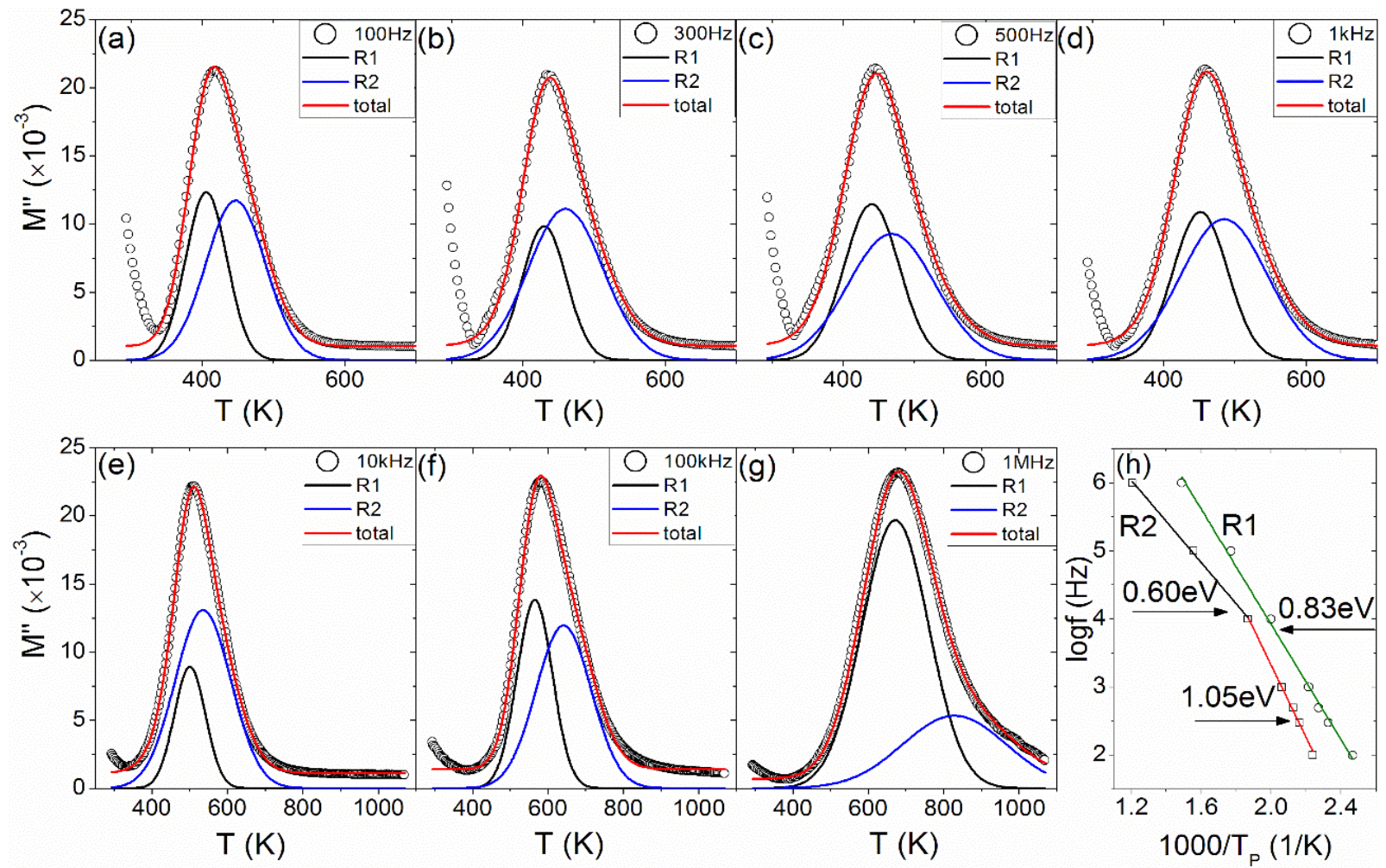

Fig. 7 Two-relaxation fittings for the electric modulus spectra of CCO (a-g) and the resultant Arrhenius plots of the relaxations (h).

energy, and $k_{B}$ is the Boltzmann constant. The relaxation vacancies migration. ${ }^{[20-24]}$ Besides, the Arrhenius plot of CCO parameters of $f_{0}$ and $E_{a}$ were calculated by linear fittings and exhibits two-segment nature. The low- $T$ segment shows an the values for all the three samples were given in Table 2. activation energy of $1.05 \mathrm{eV}$, whereas the high-Tone shows

From which one notes that the activation energy for R1 and much lower activation energy of $0.60 \mathrm{eV}$. This feature is a $\mathrm{R} 2$ lies between 0.6-1.2 eV. This binding energy is for the hallmark of oxygen vacancies transforming from hopping dielectric relaxation caused by thermally activated oxygen conduction to band conduction. ${ }^{[20]}$
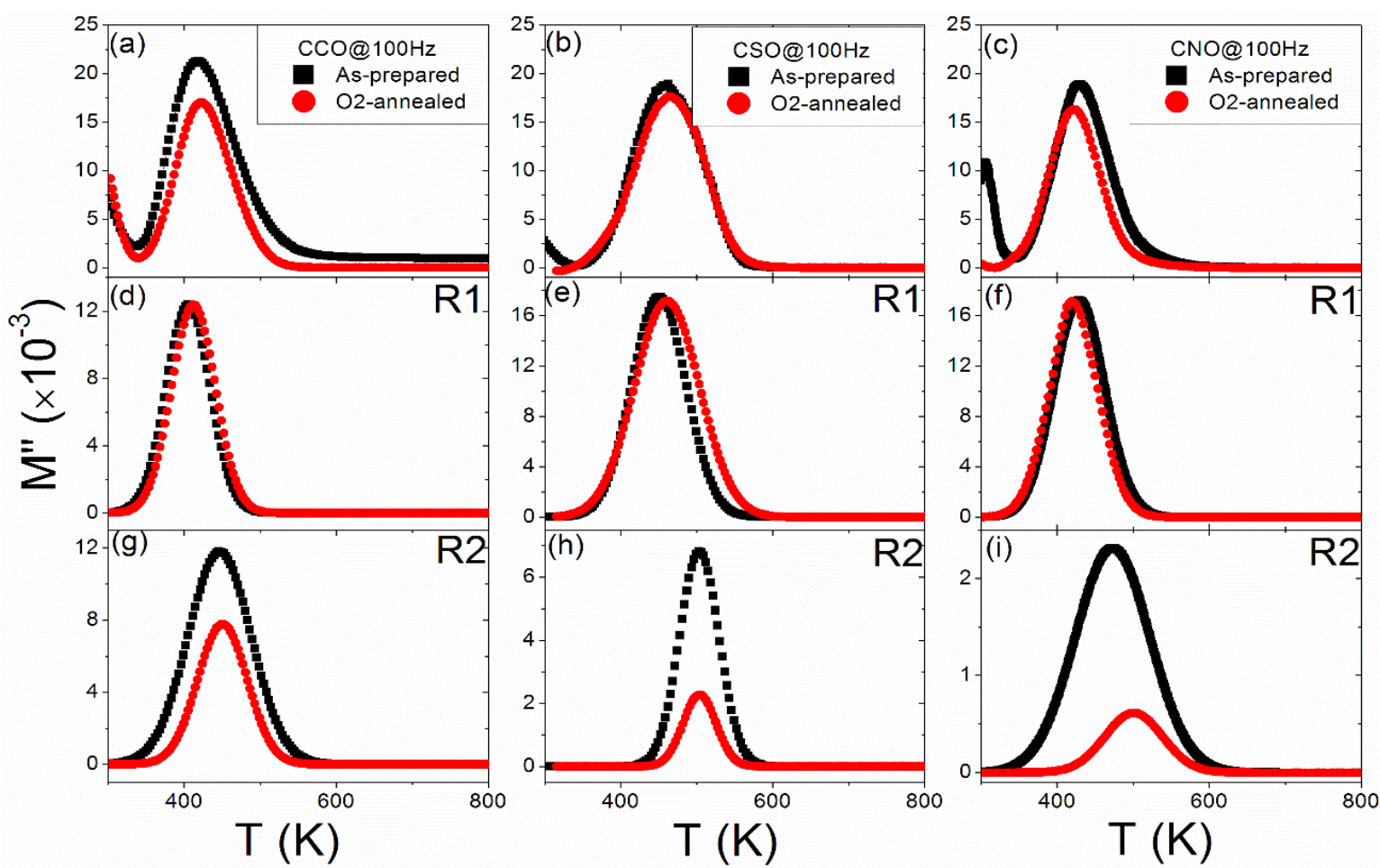

Fig. 8 Comparisons of the modulus spectra recorded at $100 \mathrm{~Hz}$ as well as the resultant $\mathrm{R} 1$ and $\mathrm{R} 2$ peaks obtained from least-square fittings between the as-prepared and $\mathrm{O}_{2}$-annealed cases for $\mathrm{CCO}(\mathrm{a}, \mathrm{d}, \mathrm{g}), \mathrm{CSO}(\mathrm{b}, \mathrm{e}, \mathrm{h})$, and $\mathrm{CNO}(\mathrm{c}, \mathrm{f}, \mathrm{i})$. 

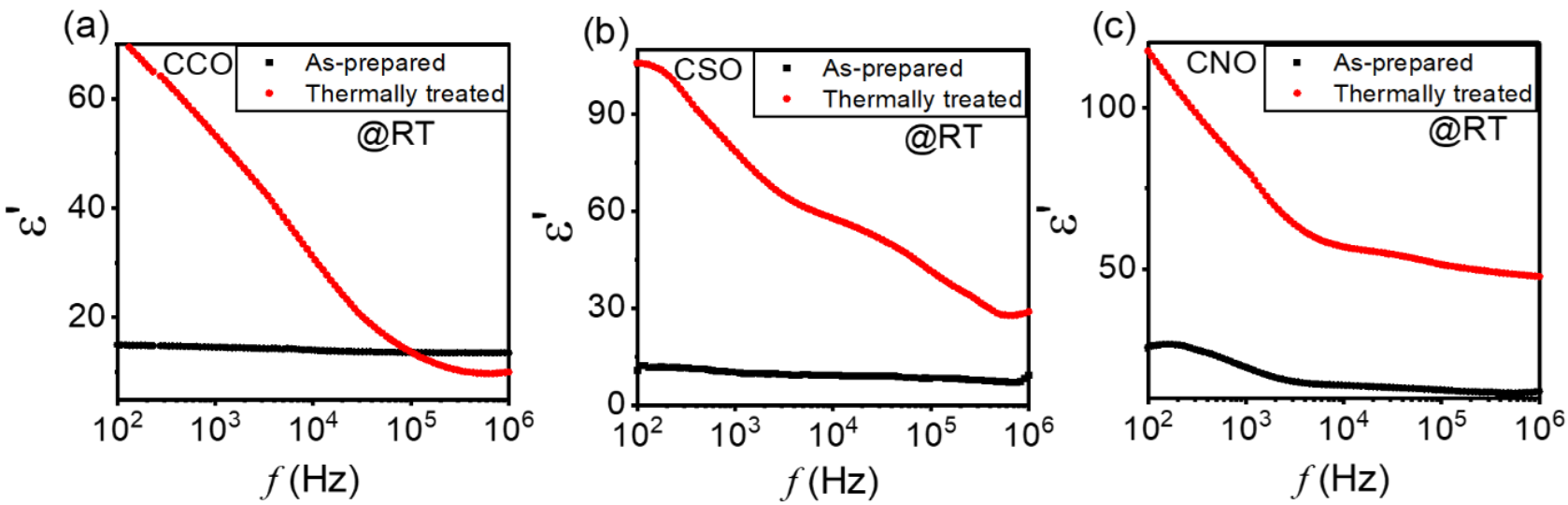

Fig. 9 Frequency dependence of the as-prepared and thermally treated samples for CCO, CSO, and CNO recorded at room temperature.

To verify this point, the as-prepared $\mathrm{CCO}, \mathrm{CSO}$, and $\mathrm{CNO}$ pellets were subjected to annealing treatments in $\mathrm{O}_{2}$ at $800{ }^{\circ} \mathrm{C}$ for $2 \mathrm{~h}$. After thermal treatment, the dielectric properties were measured as a function of temperature. Fig. 8(a), 8(b), and 8(c) show the comparison of the $M^{\prime \prime}(T)$ curves recorded at 100 $\mathrm{Hz}$ between the as-prepared and $\mathrm{O}_{2}$-annealed cases for $\mathrm{CCO}$, $\mathrm{CSO}$, and CNO, respectively. The peak comparisons of R1 and R2 detached in terms of least-squares fittings are present in Fig. 8(d)-(f) and (g)-(i), respectively. It is seen that peak R2 is depressed by the $\mathrm{O}_{2}$ annealing treatment, further confirming that this peak is related to $\mathrm{V}_{\mathrm{OS}}$. However, peak R1 is independent of the treatment. This fact evidences that $R_{1}$ has nothing to do with the $\mathrm{V}_{\mathrm{OS}}$, and therefore, the $\mathrm{V}_{\mathrm{O}}$-dopant defect associates are unlikely the origin of R1.

As mentioned earlier, the origin of $\mathrm{R} 1$ had been assigned to the vacancy-dopant defect pairs, but if this is the case, then $\mathrm{R} 1$ should be non-existent in pure ceria as well. ${ }^{[17]}$ In addition, this relaxation should have been depressed under annealing treatments. Hence, there is a need to understand the underlying phenomenon of relaxation R1. For this purpose, the samples were subjected to annealing treatments at $900{ }^{\circ} \mathrm{C}$ for $2 \mathrm{~h}$ and were air-quenched to measure the frequency-dependent dielectric properties at room temperature immediately. The annealing treatment and air-quenching ensured the complete removal of adsorbed water molecules onto the samples' surface. The results of as-prepared and thermally treated samples are shown in Fig. 9. It can be seen that the dielectric permittivity of the thermally treated samples has increased as compared to the as-prepared ones. Therein, the lowtemperature relaxation R1 was ascribed to be a MaxwellWagner relaxation caused by humidity sensitivity. To clarify this, we conducted XPS and humidity response measurements. Fig. 10 shows the capacitance curves as a function of time for the samples recorded by changing their environment $\mathrm{RH}$ level between $11 \%$ and $96 \%$. The samples' humidity response exhibits two prominent features: (1) the capacitance curves show significant change as RH level changes, further demonstrating the humidity sensitive nature of the samples. (2) the capacitance variation rapidly decreases with increasing the measurement frequency signalling the classic MaxwellWagner behaviour. ${ }^{[24]}$ Based on this feature, it follows that R1 is a Maxwell-Wagner relaxation caused by humidity sensitivity. As the samples have a pretty porous structure that promotes the surface to adsorb water molecules. These water molecules form a layer on the surface of the sample and can interact with the oxygen vacancies present there; as evident
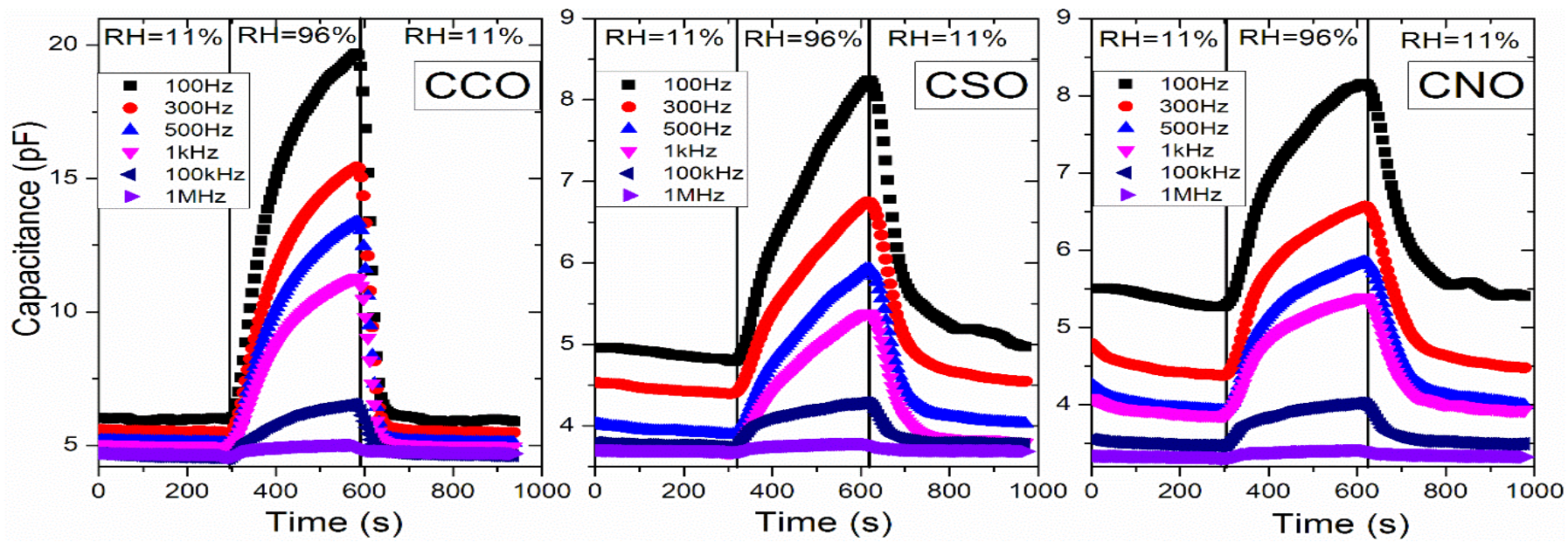

Fig. 10 Humidity responses for $\mathrm{CCO}, \mathrm{CSO}$, and $\mathrm{CNO}$ indicated by the variation of capacitance measured with different frequencies by switching the RH level between $11 \%$ and $95 \%$. 

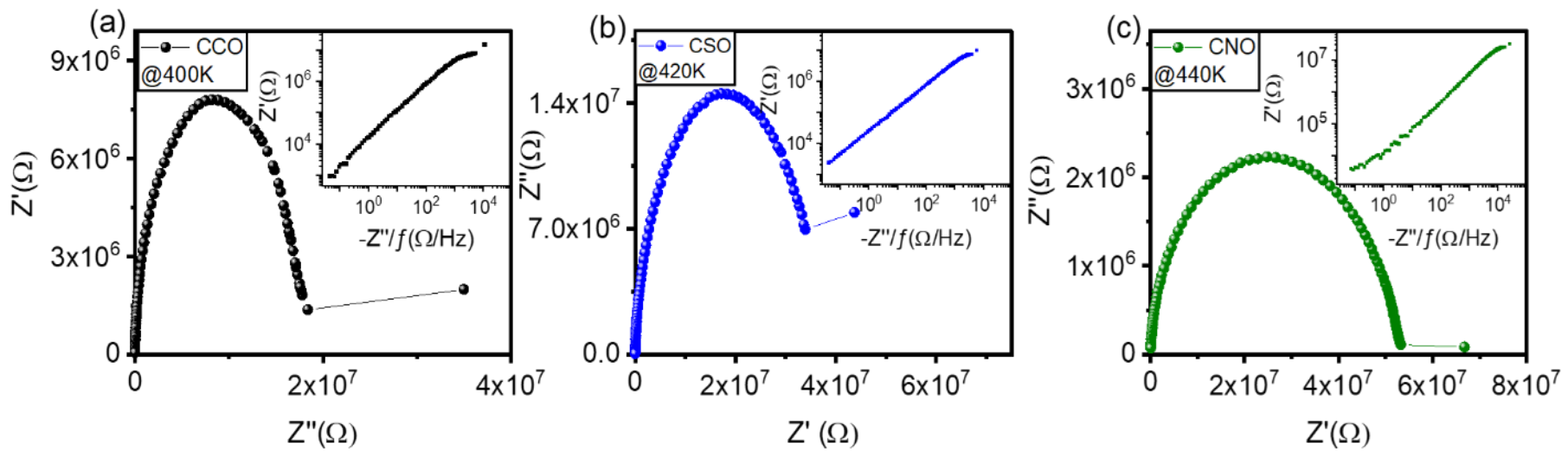

Fig. 11 Nyquist plots for (a) CCO, (b) CSO, and (c) CNO recorded at 400K, 420K and 440K, respectively. The corresponding Z' versus $Z^{\prime \prime} / f$ plots are shown in insets.

by the XPS analysis; and can generate positively charged hydroxyl ion defects $\left(\mathrm{OH}_{\mathrm{o}}^{-}\right)$. These hydroxyl ion defects combine with $\mathrm{Ce}$ ions to form $\mathrm{OH}_{\mathrm{o}}^{-}$-Ce dipoles in the samples. Under the action of external fields, these dipoles can easily reorientate and generate dipolar relaxation $\mathrm{R}_{1}$. The reduction of permittivity in thermally treated and air-quenched samples supports the above result. ${ }^{[25-28]}$

Fig. 11 shows the Cole-Cole plots of $\mathrm{CCO}, \mathrm{CSO}$ and $\mathrm{CNO}$ at 400,420 and $440 \mathrm{~K}$ temperature, respectively, as it can tell the contribution of bulk and interface towards dielectric properties. However, it is difficult to differentiate the bulk contribution from the interfacial effects solely from Cole-Cole plots. To get a better understanding, $Z^{\prime}$ versus $Z^{\prime \prime} / f$ was plotted as shown in the insets of Fig. 11. This is a powerful tool to decipher the interfacial effect information. This plot can effectively represent the effect of contacts, grain boundaries and bulk in a low to high-frequency spectrum with just the help of three straight lines. It can be seen in Fig. 11 that the graphs at the low-frequency region deviate from the straight lines. This horizontal deviation indicates the dominating effect of interfacial contribution because of the adsorbed humidity layer onto the sample's surface, giving rise to R1. This effect can also be realized in the samples' Cole-Cole plots, where two semi-circular arcs are seen with a little tail at lower frequency. ${ }^{[29-30]}$

\section{Conclusion}

A comprehensive study of the dielectric properties of $\mathrm{Ce}_{1}$. ${ }_{\mathrm{x}} \mathrm{CaxO}_{2-\delta}, \mathrm{Ce}_{1-\mathrm{x}} \mathrm{Sm}_{\mathrm{x}} \mathrm{O}_{2-\delta}$, and $\mathrm{Ce}_{1-\mathrm{x}} \mathrm{Nd}_{\mathrm{x}} \mathrm{O}_{2-\delta}$ with $\mathrm{x}=0.1,0.2$, and 0.3 , respectively, was performed. All three materials show two thermally activated relaxations labelled as R1 and R2. The $\mathrm{O}_{2-}$ annealing treatments prove that the high-temperature relaxation $\mathrm{R} 2$ results from the hopping motion of oxygen vacancies as it was depressed under the annealing treatment. While the low-temperature relaxation $\mathrm{R} 1$ was unaffected by the annealing treatment. A comparison of the dielectric properties of the as-prepared and thermally treated samples and humidity sensitivity measurements showed that R1 is a Maxwell-Wagner relaxation caused by $\mathrm{OH}_{0}^{-}$-Ce dipoles due to adsorbed humidity. The Nyquist plots of $\mathrm{CCO}, \mathrm{CSO}$ and $\mathrm{CNO}$ at 400,420 and $440 \mathrm{~K}$ also verified this finding.

\section{Acknowledgements}

The authors acknowledge financial support from the National Natural Science Foundation of China (Grant no. 51872001 and 51502001). This work was supported in part by the Open Research Fund Program of the State Key Laboratory of LowDimensional Quantum Physics (Grant no. KF201802).

\section{Supporting information}

Applicable.

\section{Conflict of interest}

There are no conflicts to declare.

\section{References}

[1] T. H. Santos, J. P. F. Grilo, F. J. A. Loureiro, D. P. Fagg, F. C. Fonseca, D. A. Macedo, Ceram. Int., 2018, 44, 2745-2751. doi: 10.1016/j.ceramint.2017.11.009.

[2] A.-K. Elger, J. Baranyai, K. Hofmann, C. Hess, Direct Operando Spectroscopic Observation of Oxygen Vacancies in Working Ceria-Based Gas Sensors, ACS Sensors, 2019. doi: 10.1021/acssensors.9b00521.

[3] F. Zhou, X. Song, X. Zhou, J. Gao, J. Bao, Z. Tian, S. An, Ceram. Int., 2019, 45, 12060-12065.doi: 10.1016/j.ceramint.2019.03.102.

[4] S.I. Ahmad, T. Mohammed, A. Bahafi, M.B. Suresh, Appl. Nanosci., 2017, 7, 243-252. doi: 10.1007/s13204-017-0567-x. [5] K. C. Anjaneya, G.P. Nayaka, J. Manjanna, G. Govindaraj, K.N. Ganesha, J. Alloy Compd., 2013, 578, 5359. doi: 10.1016/j.jallcom.2013.05.010.

[6] K. Eguchi, T. Setoguchi, T. Inoue, H. Arai, Solid State Ionics., 1992, 52, 165-172. doi: 10.1016/01672738(92)90102-U.

[7] B. Ji, C. Tian, C. Wang, T. Wu, J. Xie, M. Li, J. Power Sources., 2015, 278, 420-429, doi: 10.1016/j.jpowsour.2014.12.073.

[8] K.C. Anjaneya, G. P. Nayaka, J. Manjanna, V. M. A. Kumar, G. Govindaraj, K. N. Ganesha, J. Alloy Compd., 2014, 598, 33-40, doi: 10.1016/j.jallcom.2014.01.242. 
[9] R. Ahmed, S. ur Rehman, R. Si, C. Wang, Phys. Status Solidi B., 2021, 258, 2000342, doi:10.1002/pssb.202000342. [10] R. Ahmed, J. Wang, R.J. Si, S. ur Rehman, T. Li, H. Bi, Y. Yu, Q.J. Li, Y.D. Li, S.G. Huang, J. Eur. Ceram. Soc., 2021, 41, 2625-2632, doi: 10.1016/j.jeurceramsoc.2020.11.034.

[11] R. Ahmed, R. Si, S. ur Rehman, Y. Yu, Q. Li, C. Wang, Results Phys., 2021, 20, 103623, doi: 10.1016/j.rinp.2020.103623.

[12] R. Ahmed, R. Si, S. ur Rehman, Y. Yu, Q. Li, C. Wang, Phys. B Condens. Matter., 2021, 603, 412704, doi: 10.1016/j.physb.2020.412704.

[13] M. Burbano, S.T. Norberg, S. Hull, S.G. Eriksson, D. Marrocchelli, P.A. Madden, G.W. Watson, Chem. Mater., 2011, 24, 222-229, doi: 10.1021/cm2031152.

[14] D. Marrocchelli, P.A. Madden, S.T. Norberg, S. Hull, Chem. Mater., 2011, 23, 1365-1373, doi: 10.1021/cm102809t. [15] S. ur Rehman, H. Bi, Electrodes for Flexible Integrated Supercapacitors, Flex. Supercapacitor Nanoarchitectonics. 2021, 1-26. doi: 10.1002/9781119711469.ch1.

[16] P. Sarkar, P.S. Nicholson, J. Phys. Chem. Solids., 1989, 50, 197-206, doi: 10.1016/0022-3697(89)90418-6.

[17] J.S. Kim, J. Phys. Soc. Japan. 2001, 70, 3129-3133, doi: 10.1143/jpsj.70.3129.

[18] H. Yamamura, S. Takeda, K. Kakinuma, Solid State Ionics., 2007, 178, 1059-1064, doi: 10.1016/j.ssi.2007.05.010. [19] H. Yamamura, S. Takeda, K. Kakinuma, J. Ceram. Soc. Japan., 2007, 115, 471-474, doi: 10.2109/jcersj2.115.471.

[20] A. Hassan, M.A. Aslam, M. Bilal, M.S. Khan, S. ur Rehman, K. Ma, J. Wang, Z. Sheng, Ceram. Int. 2021, doi: 10.1016/j.ceramint.2021.04.014.

[21] C. Artini, M. Pani, M. M. Carnasciali, M. T. Buscaglia, J.R. Plaisier, G. A. Costa, Inorg. Chem., 2015, 54, 4126-4137, doi: 10.1021/acs.inorgchem.5b00395.

[22] S. ur Rehman, M. Sun, M. Xu, J. Liu, R. Ahmed, M. Adnan Aslam, R. Ali Ahmad, H. Bi, J. Colloid Interf. Sci., 2020, 574, 87-96, doi: 10.1016/j.jcis.2020.04.053.

[23] S. ur Rehman, R. Ahmed, J. Liu, J. Wang, M. Sun, Z. Fang, M.A. Aslam, P.C. Morais, C. Wang, H. Bi, Part. Syst. Charact., 2019, 36, 1900047, doi: 10.1002/ppsc.201900047.

[24] Y. Wang, Y. Liu, C. Wang, H. Liu, J. Zhang, J. Lin, J. Fan, T. Ding, J.E. Ryu, Z. Guo, Eng. Sci., 2020, 9, 50-59. doi: 10.30919/es8d903.

[25] S. ur Rehman, J. Wang, Q. Luo, M. Sun, L. Jiang, Q. Han, J. Liu, H. Bi, Chem. Eng. J., 2019, 373, 122-130. doi: 10.1016/j.cej.2019.05.040.

[26] R. Nie, Q. Wang, P. Sun, R. Wang, Q. Yuan, X. Wang, Eng. Sci., 2019, 6, 22-29, doi: 10.30919/es8d668.

[27] S. ur Rehman, R. Ahmed, K. Ma, S. Xu, T. Tao, M.A. Aslam, M. Amir, J. Wang, Eng. Sci., 2020, 13, 71-78, doi: 10.30919/es8d1263.

[28] Sajid ur Rehman, J. Liu, Z. Fang, J. Wang, R. Ahmed, C. Wang, H. Bi, ACS Appl. Nano Mater., 2019, 2, 4451-4461, doi: 10.1021/acsanm.9b00841.

[29] W. Uddin, S. ur Rehman, M.A. Aslam, S. ur Rehman, M. Wu, M. Zhu, Mater. Res. Bull., 2020, 130, 110943, doi: 10.1016/j.materresbull.2020.110943.

[30] H. Huang, L. Han, Y. Wang, Z. Yang, F. Zhu, M. Xu, Eng. Sci., 2019, 9, 60-67, doi: 10.30919/es8d812.

[31] M.C. Biesinger, B.P. Payne, A.P. Grosvenor, L.W.M. Lau, A.R. Gerson, R.S.C. Smart, Appl. Surf. Sci., 2011, 257, $2717-$ 2730, doi: 10.1016/j.apsusc.2010.10.051.

[32] R. Ahmed, S. T. Wang, J. Sun, J. Wang, T. Y. Li, Y. Yu, Q. J. Li, C. C. Wang, Ceram. Int., 2019, 45, 13484-13487, doi: 10.1016/j.ceramint.2019.04.051.

\section{Author information}

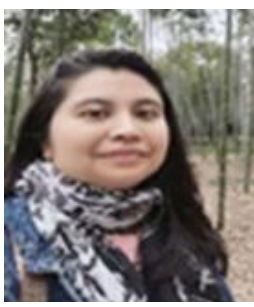

Rida Ahmed received her PhD degree in Materials Physics from Anhui University, Hefei, P. R. China. Her research is focused on dielectric materials and energy storage devices.

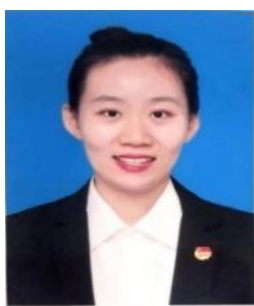

Shuting Wang received her Master's degree in Materials Physics from Anhui University, Hefei, P. R. China. Her research is focused on dielectric materials and energy storage devices

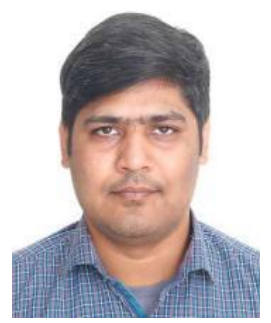

Sajid ur Rehman received his PhD degree in Polymer Chemistry and Physics from Anhui University, Hefei, China. Currently, he is on Post-doctoral position at High Magnetic Field Laboratory, Hefei Institute of Physical Sciences, Chinese Academy of Sciences, Hefei, China. His research focus is on biomaterials, energy storage devices and microwave absorption properties.

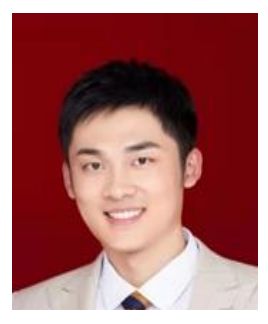

Sun Jie received his Master's degree in Materials Physics from Anhui University, Hefei, P. R. China. His research is focused on dielectric materials and energy storage devices.

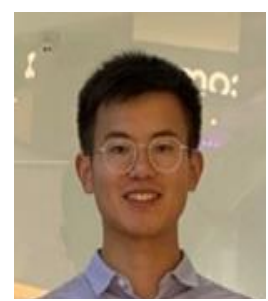

Wang Jin received his Master's degree in Materials Physics from Anhui University, Hefei, $P . R$. China. His research is focused on dielectric materials and energy storage devices. 


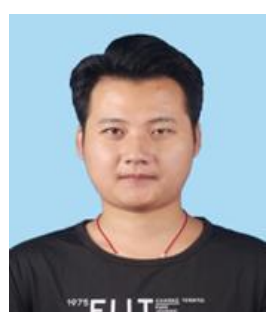

Si Renjun doing his Master's degree in Materials Physics from Anhui University, Hefei, P. R. China. His research is focused on dielectric materials and humidity sensors.

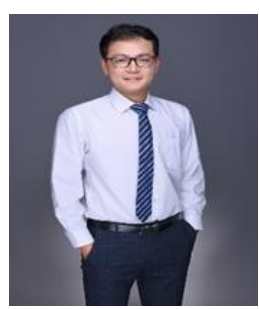

An Kang Zhu is doing his Ph.D. degree in Materials Physics from Anhui University, Hefei, P. R. China. His research is focused on solid oxide fuel cells.

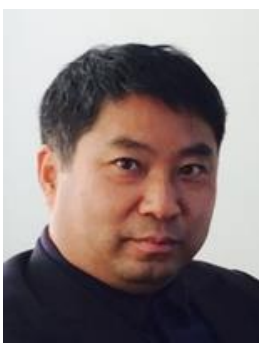

Chunchang Wang Currently working in School of Physics and Material Science, Anhui University (AHU). He received his B.S. degree from $A H U$ in 1993, M.S. degree from Institute of Solid State Physics, Chinese Academy of Sciences, in 1997, and PH. D. degree from Tsinghua University in 2004. Before joining AHU, he was a Researcher Fellow first at National Institute of Advanced Industrial Science and Technology, Japan, then at University of Wollongong, Australia. His research interests include dielectric physics and the applications of dielectric materials in the fields of energy storage, sensors, and photocatalysis.

Publisher's Note: Engineered Science Publisher remains neutral with regard to jurisdictional claims in published maps and institutional affiliations. 